\title{
Asteroseismology of massive stars with the MOST satellite
}

\author{
Anthony F. J. Moffat ${ }^{1}$ and The MOST Team \\ ${ }^{1}$ Département de physique, Université de Montréal, \\ C.P. 6128, Succursale Centre-ville, Montréal, QC, H3C 3J7, Canada \\ email: moffat@astro.umontreal.ca
}

\begin{abstract}
Since 2003 the MOST (Microvariability and Oscillations of STars) microsatellite has obtained typically a month of non-stop, minute-of-time resolution, high-precision, singlebroadband optical photometry for each of a significant number of Galactic OB and WR stars. Numerous p- and g-modes were clearly detected in several OB stars, including discovery of g-modes for the first time in a blue supergiant (Saio et al. 2006). True rotation periods were found for some SPBe pulsators (Cameron et al. 2008). Many O stars are remarkably quiet. Five presumably single WR stars have been observed so far, each interesting in its own way. In particular, the cool WR stars WR123 (WN8) and WR103 (WC9d) both show mostly short-lived, multimode oscillations with most of the Fourier power occurring on a day or longer timescale (Moffat et al. 2008a). WR123 also revealed a fairly stable 10-hour periodicity (Lefèvre et al. 2005). All of these oscillations probably arise in the stellar cores. WR111 (WC5) shows no (coherent) oscillations above the detection limit of $0.05 \mathrm{mmag}$ in the 10 -minute period range predicted for strange-mode pulsations at a level of $2 \mathrm{mmag}$ (Moffat et al. 2008b). WR110 (WN5-6 and a stronger-than-average X-ray source) and WR124 (WN8h, i.e. in contrast with the previously observed, hydrogen-free WR123 of otherwise similar subtype), both strongly variable with MOST, are currently being analyzed. The next target just observed (late-June to early Aug 2009) is the 30-day eclipsing binary CV Ser = WR113 (WC8d + O8-9IV). Besides stellar oscillations, we will also search for orbital-phase dependent, stochastic variability in $\mathrm{CV}$ Ser as wind clumps in the WR component's dense wind pass in front of the O-star.
\end{abstract}

Keywords. Space vehicles, stars: early-type, stars: oscillations (including pulsations), stars: Wolf-Rayet.

\section{References}

Cameron, C., Saio, H., Kuschnig, R., Walker, G. A. H., Matthews, J. M., Guenther, D. B., Moffat, A. F. J., Rucinski, S. M., Sasselov, D., Weiss, W. W. 2008, ApJ, 685, 489

Lefèvre, L., Marchenko, S. V., Moffat, A. F. J., Chené, A. N., Smith, S. R., St-Louis, N., Matthews, J. M., Kuschnig, R., Guenther, D. B., Poteet, C. A., Rucinski, S. M., Sasselov, D., Walker, G. A. H., Weiss, W. W. 2005, ApJ, 634, L109

Moffat, A. F. J., Marchenko, S. V., Lefèvre, L., Chené, A.-N., St-Louis, N., Zhilyaev, B. E., Aerts, C., Saio, H., Walker, G. A. H., Matthews, J. M., Kuschnig, R., Cameron, C., Rowe, J. F., Guenther, D. B., Rucinski, S. M., Sasselov, D., Weiss, W. W. 2008a, in: A. de Koter, L. J. Smith, \& L. B. F. M. Waters (eds.), ASP Conference Series (San Francisco: Astronomical Society of the Pacific), 388, 29

Moffat, A. F. J., Marchenko, S. V., Zhilyaev, B. E., Rowe, J. F., Muntean, V., Chené, A.-N., Matthews, J. M., Kuschnig, R., Guenther, D. B., Rucinski, S. M., Sasselov, D., Walker, G. A. H., Weiss, W. W., 2008b, ApJ, 679, L45

Saio, H., Kuschnig, R., Gautschy, A., Cameron, C., Walker, G. A. H., Matthews, J. M., Guenther, D. B., Moffat, A. F. J., Rucinski, S. M., Sasselov, D., Weiss, W. W. 2006, ApJ, 650, 1111 